\title{
Persistent Pleuropulmonary Air Leak Treated with Autologous Blood: Results from a University Hospital and Review of Literature
}

\author{
Frederico H.S. Oliveira Daniele C. Cataneo Raul L. Ruiz, Jr. \\ Antonio J.M. Cataneo \\ Thoracic Surgery Discipline of the Surgery and Orthopedics Department, Botucatu School of Medicine, \\ São Paulo State University, São Paulo, Brazil
}

Key Words

Blood $\cdot$ Fistula $\cdot$ Pleura $\cdot$ Pneumothorax

\begin{abstract}
Background: Persistent air leak after pulmonary resection is a difficult complication for thoracic surgeons to manage. Objectives: To show the results of our experience treating persistent pleuropulmonary air leak with autologous blood and review the literature on this specific method of treatment. Methods: Retrospective study of patients with persistent aerial pleuropulmonary fistula treated with autologous blood. The patient's own blood was collected from a peripheral vein and directly introduced through the pleural drain. An inverted siphon was located in the drainage system to avoid prolonged clamping of the drain. This siphon impeded blood return but not air escape. Results: Between January 2001 and August 2008, 27 patients were treated by the above method. Patient age ranged from 2 to 74 years, and $78 \%$ were male. Each procedure used a mean quantity of 92 $\mathrm{ml}$ blood. Mean persistent air leak time before pleurodesis was 10.6 days and mean time to fistula resolution after pleurodesis was 1.5 days. Twenty-three (85\%) patients had persistent pleuropulmonary air leak closed with the above procedure. Conclusion: Treating persistent pleuropulmonary air leak with autologous blood is promising, but further studies are required to quantify its real effectiveness.
\end{abstract}

Copyright $\odot 2009$ S. Karger AG, Basel

\section{KARGER}

Fax +4161306 1234 E-Mail karger@karger.ch www.karger.com
(C) 2009 S. Karger AG, Basel

0025-7931/10/0794-0302\$26.00/0

Accessible online at:

www.karger.com/res

\section{Introduction}

Persistent pleuropulmonary air leaks are extremely distressing to the thoracic surgeon. Their origin can be classified as primary spontaneous pneumothorax (without coexisting pulmonary disease) or secondary (associated with underlying pathology) [1]. The latter is very common and difficult to manage. There are several procedures for treating persistent air leak, the most suitable being chosen on a patient-by-patient basis. Different types of sclerosant used in pleurodesis [2] include talc [3-6], antibiotics and antineoplastics [7-9] and autologous blood $[10,11]$. The surgical procedures include thoracoplasty, myoplasty, open pleural drainage, control by suturing or occlusion with biological agent [12].

Autologous blood was originally used to avoid relapse of spontaneous primary pneumothorax [10] and its efficacy in treating persistent air leak was later verified [13]. Fibrin formed from the fibrinogen contained in blood does not provide firm fixing for the pleura, and is not indicated for treatment in pleural effusion, but seems to be adequate for aerial fistula occlusion.

The objective of this study is to show the results of treating persistent pleuropulmonary air leak with autologous blood and review literature on this specific method of treatment.
Antonio José Maria Cataneo

Thoracic Surgery Discipline of the Surgery and Orthopedics Department

Botucatu School of Medicine, São Paulo State University, UNESP

18618-970 Botucatu, SP (Brazil)

Tel. +55 143815 6230, Fax +55 143815 7615, E-Mail acataneo@ fmb.unesp.br 


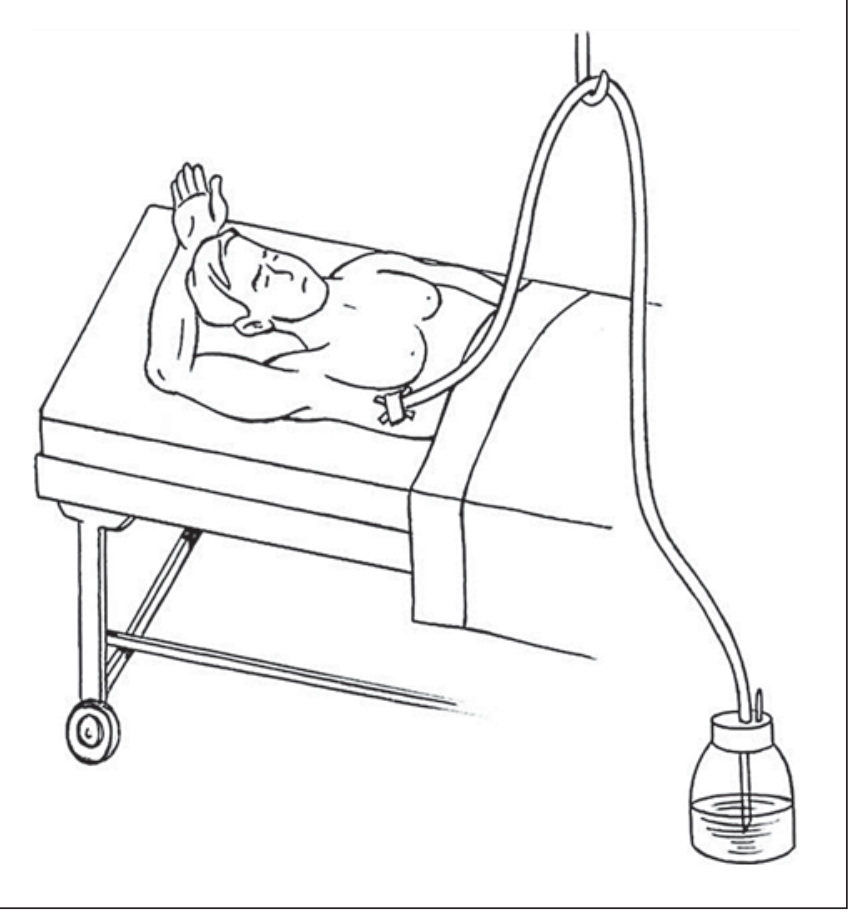

Fig. 1. Inverted siphon prevents blood returning down the drain.

\section{Patients and Methods}

After approval from the São Paulo State University's research ethics committee, a review was started of medical records from patients treated with autologous blood, from 2001, when the procedure was introduced, to August 2008.

Studied Population. All cases of persistent air leak with five days or more duration, treated with autologous blood. Patients were excluded if they had been submitted to previous treatment with other sclerosant agents.

Collection and Application of Blood in the Pleural Cavity. Autologous blood was collected from the arm peripheral vein by 20 $\mathrm{ml}$ disposable syringe without heparin and with all the care necessary to maintain asepsis and anti-sepsis for the procedure. Blood volumes collected varied in aliquots of about $20 \mathrm{ml}$ and according to free pleural cavity size, the blood was introduced until it was full, looking to not exceed $200 \mathrm{ml}$ per session, even if it had not completely filled the cavity. While one operator collected the blood, another clamped the drain, disconnected and performed external anti-sepsis on the drain. Next, each syringe with collected blood was connected to the thoracic drain, this was then unclamped and the blood introduced into the pleural cavity. So that blood would not return down the drain and not clamp it due to the fistula, an inverted siphon was developed (fig. 1) connected to the flask from water seal. Initially we left the siphon for $24 \mathrm{~h}$, but we have reduced this time and currently we leave it for only $30 \mathrm{~min}$, but sufficient for coagulation cascade to occur and liquid to be drained, then traditional drainage was resumed. If after 2-3 days air is still leaking, the procedure is repeated. Treatment is considered to have failed when there is no reduction in bubbling, and is successful when the air leak stops totally.

Patients remained in hospital until there was no more bubbling by the drain and its removal was possible. Complications such as fever, pain and infection were also evaluated. Inpatient and outpatient evolution follow-up was made by thorax X-ray.

\section{Results}

Twenty-seven persistent pleuropulmonary air leak patients were treated with pleurodesis using autologous blood from January 2001 until August 2008. Mean age was 35 years (range 2-74). Twenty-one patients (78\%) were male and $6(22 \%)$ female. The most common causes of persistent air leak were pulmonary resection in 10 and spontaneous pneumothorax in 9 patients. The right hemithorax was affected more often (20 patients), than the left (7 patients). Depending on disease from which the fistula originated, the number of drains were 1 (13 cases) or 2 (14 cases). Mean drainage time before blood pleurodesis was 10.6 days and mean aerial fistula closing time after the procedure was 1.5 days (table 1 ).

Infused blood volume varied from 24 to $200 \mathrm{ml}$ per session (mean $92 \mathrm{ml}$ ). The process needed repeating in 7 patients. Three evolved to open pleural drainage due to failure of autologous blood treatment.

Of the 20 patients who underwent a single session, 18 were successful and 2 not. One of the 2 (P11) had been reoperated because the resection edge was compromised by neoplasia. After bronchoplasty to remove the upper right bronchial lobe stump, the patient presented persistent air leak, without having dehiscence confirmed by bronchoscopy. As the first session with autologous blood showed no improvement, open drainage was chosen. The other patient (P12) also showed no improvement, and as it was a pneumothorax secondary to emphysema disease, we preferred to perform thoracoscopy for blebs clipping and abrasive pleurodesis. In the 6 cases where 2 sessions were performed, 5 had partial resolution in the first and total resolution in the second session, and the 1 case which did not resolve had suffered decortication (P17) and probably had air leaks over the whole pleural surface. We decided to discharge this patient with an open drain. The only patient where we tried 4 sessions without success (P6) was aged, emphysematous and not in a condition to undergo surgery. Each session produced an excellent improvement in air leaks, but bubbling came back again. In the end we opted for talc instillation and open drain. 
Table 1. Patients (P) submitted to autologous blood pleurodesis, air leak origin, drainage time before pleurodesis in days $(\mathrm{T})$, total number of sessions performed $(\mathrm{N})$, time in days between final pleurodesis and bubble cessation $(\mathrm{t})$, and success $(\mathrm{S})$

\begin{tabular}{|c|c|c|c|c|c|}
\hline $\mathrm{P}$ & Air leak origin & $\mathrm{T}$ & $\mathrm{N}$ & $\mathrm{t}$ & S \\
\hline 1 & Primary spontaneous pneumothorax & 13 & 2 & 1 & yes \\
\hline 2 & Post lobectomy & 12 & 1 & 1 & yes \\
\hline 3 & Empyema & 15 & 1 & 1 & yes \\
\hline 4 & Post pulmonary biopsy & 14 & 1 & 1 & yes \\
\hline 5 & Post lobectomy & 11 & 1 & 1 & yes \\
\hline 6 & Secondary spontaneous pneumothorax & 14 & 4 & - & no \\
\hline 7 & Primary spontaneous pneumothorax & 20 & 2 & 1 & yes \\
\hline 8 & Post lobectomy & 11 & 1 & 1 & yes \\
\hline 9 & Primary spontaneous pneumothorax & 13 & 1 & 1 & yes \\
\hline 10 & Post bullectomy & 8 & 1 & 2 & yes \\
\hline 11 & Post lobectomy & 5 & 1 & - & no \\
\hline 12 & Secondary spontaneous pneumothorax & 11 & 1 & - & no \\
\hline 13 & Secondary spontaneous pneumothorax & 5 & 1 & 3 & yes \\
\hline 14 & Post bullectomy & 11 & 1 & 2 & yes \\
\hline 15 & Primary spontaneous pneumothorax & 5 & 1 & 2 & yes \\
\hline 16 & Post bullectomy & 6 & 1 & 3 & yes \\
\hline 17 & Post decortication & 34 & 2 & - & no \\
\hline 18 & Post lobectomy & 8 & 1 & 1 & yes \\
\hline 19 & Post lobectomy & 12 & 1 & 3 & yes \\
\hline 20 & Post lobectomy & 9 & 1 & 1 & yes \\
\hline 21 & Post decortication & 14 & 1 & 1 & yes \\
\hline 22 & Post bullectomy & 6 & 2 & 1 & yes \\
\hline 23 & Post lobectomy & 10 & 2 & 2 & yes \\
\hline 24 & Primary spontaneous pneumothorax & 6 & 1 & 1 & yes \\
\hline 25 & Primary spontaneous pneumothorax & 5 & 2 & 2 & yes \\
\hline 26 & Post lobectomy & 5 & 1 & 1 & yes \\
\hline 27 & Post lobectomy + rib resection & 7 & 1 & 2 & yes \\
\hline
\end{tabular}

One patient developed empyema after pleural cavity blood infusion, and 2 patients with air leak after empyema were submitted to autologous blood treatment. Another patient presented fever as a complication from the procedure.

The success rate for pleurodesis with autologous blood was $85 \%$, being effective in 23 out of 27 patients treated (table 1).

\section{Discussion}

Persistent pleuropulmonary air leak is a serious, sometimes fatal, condition which can occur after pulmonary surgery or as a complication of a subjacent pulmonary disease [14]. Some of the techniques for treating persistent pleuropulmonary fistula are well established in thoracic surgery. These techniques include surgical closing of the fistula, open pleural drainage, thoracoplasty, myoplasty and pleurodesis using various sclerosant substances such as talc, tetracycline or bleomycin $[15,16]$.

Andrés et al. [13] treated 6 persistent air leak patients with autologous blood with $100 \%$ success $24 \mathrm{~h}$ after the procedure. However, Robinson [10] only managed $84 \%$ success with the same treatment in 25 pneumothorax relapse patients. In a total of 167 spontaneous pneumothorax patients, Cagirici et al. [1] treated 32 patients with autologous blood, with an $84 \%$ success rate. When compared to other patients submitted to simple thoracic drainage (135 patients), air leak loss was significantly less in those submitted to autologous blood ( $\mathrm{p}<0.01)$. Blanco Blanco et al. [17], following 17 patients for more than a year, had an immediate $71 \%$ success rate treating spontaneous pneumothorax with autologous blood.

Andreetti et al. [18] showed that treating persistent air leak patients for more than 6 days after lobectomy with $100 \mathrm{ml}$ autologous blood was significantly better than treating them with $50 \mathrm{ml}$, and better than not treating them with blood. Our study did not aim to quantify blood volume used, but apparently the quantity used did not influence our results.

Droghetti et al. [19] treated 21 persistent air leak patients with autologous blood after pulmonary resection and obtained an $81 \%$ resolution rate in the first $24 \mathrm{~h}$. The rest underwent a new pleurodesis $36 \mathrm{~h}$ after the first which was successful. Ando et al. [20] also reported successfully closing persistent aerial fistulas with autologous blood even with incomplete lung expansion.

Shackcloth et al. [21], in a prospective randomized controlled trial, treated prolonged air leak after lobectomy with intrapleural instillation of autologous blood. The control group continued to be treated by tube thoracostomy alone. They concluded that this technique is effective in sealing air leaks after lobectomy. It allows earlier chest drain removal and shortens hospital stay.

Martínez-Escobar et al. [22] studied patients with acute respiratory distress syndrome accompanied by persistent aerial pneumothorax fistula. They had 2 groups, 1 treated with autologous blood and the other with other treatments. They concluded that autologous blood reduced mechanical ventilation weaning time by quicker air leak resolution. Williams and Laing [23] reported a case of cystic fibrosis with secondary pneumothorax which was successfully treated by autologous blood pleurodesis. Chadwick et al. [24] also reported a case of bronchiolitis obliterans which evolved with pneumothorax and air leak, and was successfully treated with autologous blood. 
Lang-Lazdunski and Coonar [25] achieved 100\% success rate in treating 11 pulmonary resection patients with persistent air leak for more than 7 days after surgery with autologous blood. Although these authors reported excluding patients with positive pleural liquid culture, we did not request this examination, and in our sample we successfully used autologous blood to close air leak in 2 pleural empyema patients.

Lang et al. [26] used autologous blood clot seal to prevent pneumothorax at CT-guided lung biopsy. They concluded that the use of autologous blood clot seal placed through the coaxial sheath is strongly recommended when performing biopsy of deep lesions because it significantly reduces the frequency of pneumothorax and the subsequent need for chest tube placement and hospitalization.

Pleurodesis with autologous blood has been successfully used in aerial fistulas but has not been applied in the hydrothorax as the weak fibrin net does not produce sufficient pleurodesis [27] and does not impede liquid exudation in the pleural space. We did not find any clinical trials comparing various sclerosant agents with autologous blood in hydrothorax, but Yilmaz et al. [28] taking advantage of the idea of pleurodesis in pleural infusions, compared talc, rifampicine, and autologous blood in hydrocele sclerosis, and concluded that autologous blood is a weak sclerosant agent.

From the experience gained in these cases, for adults, in each session we can introduce up to $200 \mathrm{ml}$ of blood into the pleural cavity, or smaller aliquots if unable to introduce the whole amount. The procedure can be repeated 2 days later if bubbling still continues. We did not perform a second instillation in cases where there was no reduction in any blistering with the first instillation, but believe that we could have attempted to.

We concluded that treating persistent pleuropulmonary air leak with autologous blood is very effective, has no relevant side effects, and is very promising. However, further studies are required to quantify its real effectiveness.

\section{Acknowledgment}

We thank the Research Support Group of the Botucatu School of Medicine, São Paulo State University, UNESP, for language revision.

\section{References}

$>1$ Cagirici U, Sahin A, Cakan H, Kayabas H, Buduneli T: Autologous blood patch pleurodesis in spontaneous pneumothorax with persistent air leak. Scand Cardiovasc J 1998; 32:75-78.

2 Jantz MA, Antony VB: Pathophysiology of the pleura. Respiration 2008;75:121-133.

$\checkmark 3$ Adler RH: A talc power aerosol method for the prevention of recurrent spontaneous pneumothorax. Ann Thorac Surg 1968;5: 474-477.

4 Milanez JRC, Vargas FS, Filomeno LTB, Fernandez A, Jatene A, Light RW: Intrapleural talc for the prevention or recurrent pneumothorax. Chest 1994;106:1162-1165.

$\checkmark 5$ Thomas R, Christopher DJ, Roy A, Rose A, Chandy ST, Cherian RA, Rima J: Chilothorax following innominate vein thrombosis: a rare complication of transvenous pacemaker implantation. Respiration 2007;74:338-340.

-6 Froudarakis ME: Diagnostic work-up of pleural effusions. Respiration 2008;75:4-13.

$\checkmark 7$ Macoviak JA, Stephenson LW, Ochs R, Edmunds LH Jr: Tetracycline pleurodesis during active pulmonary pleural air leak for prevention of recurrent pneumothorax. Chest $1982 ; 81: 78-81$
8 Sassoon CSH, Light RW, Vargas FS, Gruer SE, Wang NS: Temporal evolution of pleural fibrosis induced by intrapleural minocycline injection. Am J Respir Crit Care Med 1995; 151:791-794.

9 Ciftci AO, Santialp I, Tanyel FC, Büyükpamukçu N: The association of pulmonary lymphangioleiomyomatosis with renal and hepatic angiomylipomas in a prepubertal girl: a previously unreported entity. Respiration 2007;74:335-337.

10 Robinson CL: Autologous blood for pleurodesis in recurrent and chronic spontaneous pneumothorax. Can J Surg 1987;30:428429.

11 Dumire R, Crobbe MM, Mappin FG, FontenelleLJ:Autologous 'blood patch'pleurodesis for persistent pulmonary air leak. Chest 1992;101:64-66

12 Kinoshita T, Miyoshi S, Katoh M, Yoshimasu T, Juri M, Maebeya S, Naito Y: Intrapleural administration of a large amount of diluted fibrin glue for intractable pneumothorax. Chest 2000;117:790-795.

13 Andrés JJR, Blanco S, De la Torre M: Postsurgical pleurodesis with autologous blood in patients with persistent air leak. Ann Thorac Surg 2000;70:270-272.
14 Light RW: Doenças pleurais, ed 3, 2001.

15 Adler RH, Sayek I: Treatment malignant pleural effusion: method using tube thoracostomy and talc. Ann Thorac Surg 1976;22: 8-15

16 Alfageme I, Moreno L, Huetas C, Vargas A, Hernandez J, Beiztteggui A: Spontaneous pheneumothorax: long-term results with tetracycline pleurodesis. Chest 1994;106: 347-350.

- 17 Blanco Blanco I, Sala Blanco J, Canto Argiz H, Carro del Camino F, Gorostidi Pérez J: Pleurodesis con sangre autólogo: resultados de uma serie de 17 casos com más de um ano de seguimento. Rev Clin Esp 1997;197:406410 .

18 Andreetti C, Venuta F, Anile M, De Giacomo T, Diso D, Di Stasio M, Rendina EA, Coloni GF: Pleurodesis with an autologous blood patch to prevent persistent air leaks after lobectomy. J Thorac Cardiovasc Surg 2007; 133:759-762

19 Droghetti A, Schiavini A, Muriana P, Comel A, De Donno G, Beccaria M, Canneto B, Sturani C, Muriana G: Autologous blood patch in persistent air leaks after pulmonary resection. J Thorac Cardiovasc Surg 2006; 132:556-559. 
-20 Ando M, Yamamoto M, Kitagawa C, Kumazawa A, Sato M, Shima K, Watanabe A, Shimokata K, Hasegawa Y: Autologous blood-patch pleurodesis for secondary spontaneous pneumothorax with persistent air leak. Resp Med 1999;93:432-434.

-21 Shackcloth MJ, Poullis M, Jackson M, Soorae A, Page RD: Intrapleural instillation of autologous blood in the treatment of prolonged air leak after lobectomy: a prospective randomized controlled trial. Ann Thorac Surg 2006;82:1052-1056.

-22 Martínez-Escobar S, Ruiz-Bailén M, Lorente-Acosta MJ: Pleurodesis using autologous blood: a new concept in the management of persistent air leak in acute respiratory distress syndrome. J Crit Care 2006;21: 209-216.
23 Williams P, Laing R: Tension pneumothorax complicating autologous blood patch pleurodesis. Thorax 2005;60:1066-1067.

24 Chadwick C, Marven SM, Vora AJ: Autologous blood pleurodesis for pneumothorax complicating graft-versus-host disease-related bronchiolitis obliterans. Bone Marrow Transplant 2004;33:451-453.

25 Lang-Lazdunski L, Coonar AS: A prospective study of autologous 'blood patch' pleurodesis for persistent air leak after pulmonary resection. Eur J Cardiothorac Surg 2004;26:897-900.
26 Lang EK, Ghavami R, Schreiner VC, Archilbald S, Ramirez J: Autologous blood clod seal to prevent pneumothorax at CT-guided lung biopsy. Radiology 2000;216:93-96.

27 Mitchem RE, Herndon BL, Fiorella RM, Molteni A, Battie CN, Reisz GR: Pleurodesis by autologous blood, doxycycline and talc in rabbit model. Ann Thorac Surg 1999;67:917921.

28 Yilmaz U, Ekmekçioglu O, Tatlisen A, Demirci D: Does pleurodesis for pleural effusions give bright ideas about the agents for hydrocele sclerotherapy? Int Urol Nephrol 2000;32:89-92. 